\title{
Estimación de evapotranspiración con imágenes de PROBA-V de un cultivo de sandía en la costa de Hermosillo, Sonora, México
}

\author{
Estimates of evapotranspiration with PROBA-V images in a crop field on the coast \\ of Hermosillo, Sonora, Mexico
}

\section{Ramón Saiz Rodríguez" ${ }^{1:}$ Génesis Luisana Aguirre López ${ }^{1}$, Julio Cesar Rodríguez², Christopher John Watts Thorp ${ }^{3}$, Juan Alejandro Saiz Rodríguez, Alfredo Ochoa Granillo ${ }^{4}$ y Juan Arcadio Saiz Hernández}

\footnotetext{
${ }^{1}$ Maestría en Ciencias en Geología. Universidad de Sonora. Blvd Luis Encinas y Rosales SN, Centro. 83000 Hermosillo, Sonora, México.

‡Autor responsable (ramon.saiz@unison.mx)

${ }^{2}$ Profesor del Departamento de Agricultura y Ganadería. Universidad de Sonora. Km 20.5 Carretera Hermosillo-Bahía Kino, Apartado Postal 305. Hermosillo, Sonora, México.

${ }^{3}$ Profesor del Departamento de Física, ${ }^{4}$ Profesor del Departamento de Geología, ${ }^{5}$ Profesor del Departamento de Ingeniería Civil y Minas. Universidad de Sonora. Blvd Luis Encinas y Rosales SN, Centro, 83000, Hermosillo, Sonora, México.
}

\section{RESUMEN}

La evapotranspiración es uno de los procesos del ciclo hidrológico de mayor relevancia en estudios de balance y disponibilidad de agua en las áreas agrícolas de la región árida del Noroeste de México. En este trabajo, se utilizaron imágenes del Índice de Vegetación de la Diferencia Normalizada (NDVI) obtenidas a partir del sistema satelital PROBA-V para elaborar un modelo y estimar la evapotranspiración (ET) de un cultivo de sandía en la zona agrícola de la Costa de Hermosillo, Sonora. El procedimiento se basó en la correlación entre el coeficiente de cultivo (Kc) y el valor del NDVI. Con el modelo se estimó la ET para el cultivo de sandía, los resultados se contrastaron con valores de ET medidos con el método de covarianza de vórtices. La correlación entre los valores medidos y los estimados fue de $\mathrm{r}^{2}=0.729 \mathrm{y}$, aunque los resultados fueron aceptables, pueden ser mejorados a medida que se incremente el número de imágenes satelitales de buena calidad y la ET sea medida en una mayor variedad de cultivos.

Palabras claves: covarianza de vórtices, percepción remota, Costa de Hermosillo.

Cita recomendada:

Saiz Rodríguez, R., G. L. Aguirre López, J. C. Rodríguez, C. J. Watts Thorp, J. A. Saiz Rodríguez, A. Ochoa Granillo y J. A. Saiz Hernández. 2017. Estimación de evapotranspiración con imágenes de PROBA-V de un cultivo de sandía en la costa de Hermosillo, Sonora, México. Terra Latinoamericana 35: 301-308.

\section{SUMMARY}

Evapotranspiration is one of the most important hydrological cycle processes in balance and water availability studies in a crop field of the arid region of northwestern Mexico. In this work, images of the Normalized Difference Vegetation Index (NDVI) obtained from the PROBA-V satellite were used to develop a model to estimate the evapotranspiration (ET) in a watermelon crop in an agricultural area of the coast of Hermosillo, Sonora. The procedure was based on the correlation between the crop coefficient (Kc) and the NDVI value. With the developed model, the ET of a watermelon crop was estimated and the results were contrasted with ET values measured with the eddy covariance method. The correlation between the measured values and the estimated values was $r^{2}=0.729$ and, although the results were acceptable, they can improve as the number of good quality satellite images increases and ET is measured in a greater variety of agricultural crops.

Index words: eddy covariance, remote sensing, Hermosillo Coast.

Recibido: junio de 2017. Aceptado: agosto de 2017. Publicado en Terra Latinoamericana 35: 301-308. 


\section{INTRODUCCIÓN}

La evapotranspiración (ET) es uno de los principales componentes del balance hidrológico en cuencas con vegetación natural, zonas agrícolas $\mathrm{y}$, en los últimos años, con los avances tecnológicos y el desarrollo de nuevas metodologías de análisis, su estimación es fundamental para estudios ambientales, modelos climáticos y meteorológicos (Shuttleworth, 2007; Polhamus et al., 2013).

Las variables meteorológicas como la radiación solar, temperatura del aire, humedad y vientos, generan una alta demanda de vapor de agua atmosférico en las regiones áridas del noroeste de México (Rodríguez et al., 2011) $)^{1}$, influyendo notablemente en la disponibilidad de agua y con ello limitando el desarrollo de las áreas agrícolas (Watts et al., 1999), por lo que es fundamental cuantificar apropiadamente la evapotranspiración (ET) de una forma simple, económica y que permita resultados consistentes con mediciones de superficie.

La ET, constituida por la lámina de agua que retorna a la atmósfera mediante los procesos de evaporación de superficies evaporantes y la transpiración a través de las plantas (Allen, 2006), ha sido estimada durante décadas mediante métodos que se basan en variables meteorológicas medidas de manera puntual en estaciones climatológicas (Brutsaert, 1982; Parlange et al., 1995; Jiang e Islam, 1999; Allen, 2000). Algunos utilizan parámetros determinados experimentalmente y fórmulas sin sustento científico sólido (Parlange et al., 1995), lo que resulta en determinaciones poco precisas y no aplicables para todo sitio de estudio; además, para estimar la ET en áreas extensas con vegetación, es necesario hacer muchas medidas puntuales con alguno de estos métodos y después aplicar un método de interpolación.

En las últimas décadas, el conocimiento del proceso de ET y los avances en el desarrollo tecnológico han dado lugar al desarrollo de modelos con los que se representa o estima de manera precisa el proceso de la ET. Entre los métodos basados en micrometeorología, destaca el de covarianza de vórtices, que ha sido probado con éxito en áreas pequeñas y grandes (Burba, 2013), en vegetación natural de zonas áridas en planicies y montañas (Vivoni et al., 2010; Mendez Barrozo et al., 2014) y en cultivos de hortalizas (Rodríguez et al., 2011) ${ }^{1}$ y perennes (Rodríguez et al., 2010).
Las estaciones micrometeorológicas, además de proveer datos indispensables para los programas de riego y fundamentales para estudios de balance energético en sitios agrícolas y de vegetación natural, han sido de gran utilidad para validar métodos o contrastar estimaciones de ET en grandes superficies de terreno, que se han realizado con imágenes de $1 \mathrm{~km}$ del sensor avanzado de muy alta resolución (AVHRR) de los satélites NOAA (Jiang e Islam, 1999) y con combinaciones de datos de AVHRR y del espectroradiómetro de imágenes de resolución moderada (MODIS) que portan las plataformas de sistemas satelitales AQUA y TERRA (Batra et al., 2006; Ma et al., 2014). Las imágenes de alta resolución espacial también han mostrado su utilidad para estimar ET; se han utilizado imágenes del satélite LANDSAT 8 (TIRS, VNIR), con píxeles de $100 \times 100 \mathrm{~m}$ en cuencas hidrológicas de gran extensión (Senay et al., 2016) y se han combinado con datos de MODIS, con las que se han desarrollado modelos para determinar la evapotranspiración a escala diaria (Yang et al., 2017). Los satélites SPOT-4 y SPOT-5 también dan buenos resultados en estimaciones de evapotranspiración de cultivos (Farg et al., 2012; Bisquert et al., 2016) y con el lanzamiento del satélite PROBA-V (Project for OnBoard Autonomy - Vegetation), que entró en operación en la primavera de 2013, se ofrecen productos con resolución espacial en el rango de 0.10 a $1 \mathrm{~km}$ con lo que se preserva el legado de 19 años del instrumento Vegetation que portan los satélites SPOT-4 y SPOT-5 (Dierckx et al., 2014; Francois et al., 2014) y se amplía la capacidad y calidad del sistema para estudios científicos y el desarrollo tecnológico.

El objetivo de este trabajo fue elaborar y validar un modelo a partir de la correlación lineal de valores del índice de vegetación de la diferencia normalizada (NDVI) obtenidas con datos del sistema satelital PROBA-V con datos de evapotranspiración medidos mediante el método de Covarianza de Vórtices.

\section{MATERIALES Y MÉTODOS}

\section{Área de Estudio}

El sitio de estudio comprendió el área agrícola localizada en el kilómetro 21 de la Carretera SON-100, de la Costa de Hermosillo, Sonora, México (Figura 1),

\footnotetext{
${ }^{1}$ Rodríguez, J. C., C. J. Watts, J. Garatuza-Payán, C. Lizarraga-Celaya, J. Grajeda-Grajeda, A. Ochoa-Meza, S. F. Moreno-Salazar y M. E. Rentería Martínez. 2011. Evapotranspiración y balance hídrico en nogal pecanero (Carya illinoinensis K. Koch) en la Costa de Hermosillo, México. pp. 692-698. In: Memorias XIV Congreso Internacional en Ciencias Agrícolas.
} 

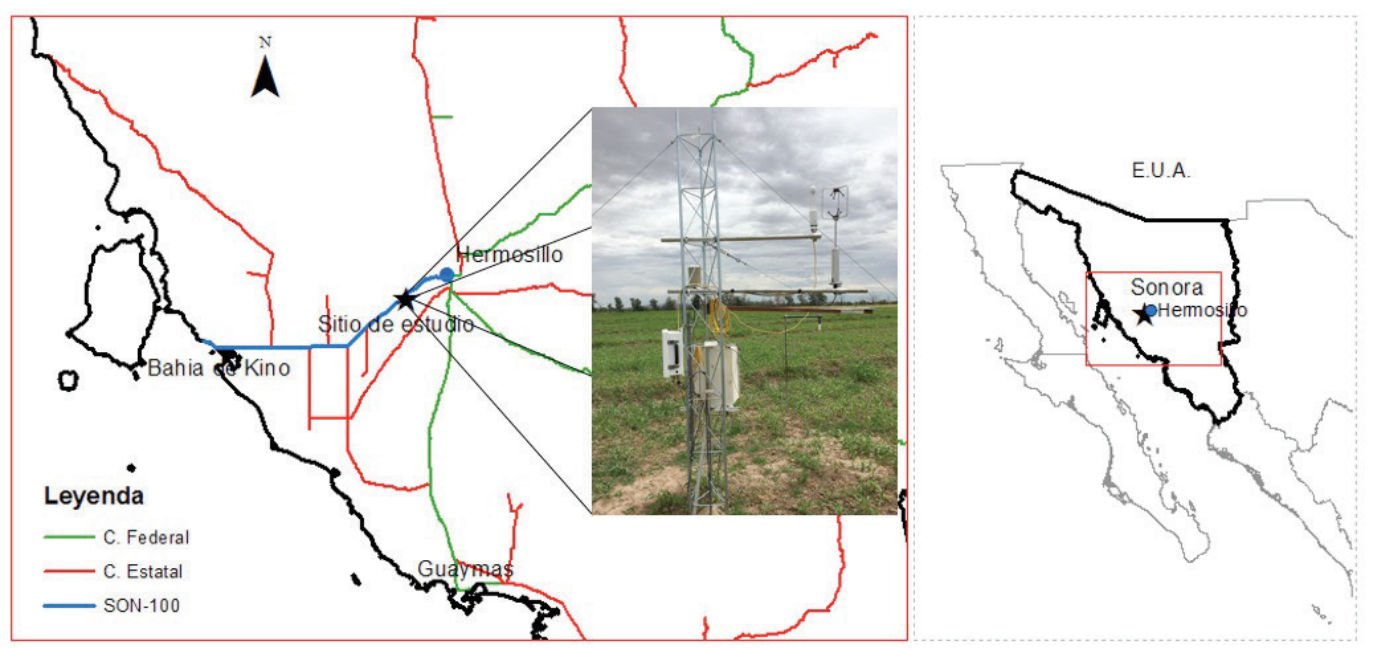

Figura 1. Localización del sitio de estudio.

en el que prevalece un clima desértico $(\mathrm{BWw})$ con precipitación y temperatura media anual de $320.8 \mathrm{~mm}$ y $23.1{ }^{\circ} \mathrm{C}$, respectivamente; la textura del suelo se caracteriza por un contenido de $49.08 \%$ de arena, $32.12 \%$ de limo y $18.8 \%$ de arcillas.

\section{Cultivos en el Área de Estudio}

El cultivo en el año 2014, fue sandía (Citrullus lanatus) mini triploide, variedad Extazy (Hazera Genetics) sobre una superficie de 60 ha, trasplantada entre el 8 y 15 de agosto, en un sistema de plantación con equidistancia entre cama de $2 \mathrm{~m}$ y longitud de $150 \mathrm{~m}$, con una densidad de 12000 plantas por ha y una secuencia de un macho cada dos hembras, 9 líneas y una calle de cosecha. Se regó con un gasto de $1.287 \mathrm{~L} \mathrm{~h}^{-1}$, con una distancia entre goteros de $0.406 \mathrm{~m}$. La cosecha de los frutos inició el 9 de octubre y finalizó el 4 de noviembre.

El análisis del año 2015, se llevó a cabo con sandía de la variedad Fantasy, sembrada en una superficie de 120 ha, trasplantada entre el 20 y 21 de enero y plantados con la misma configuración y características que el cultivo del año 2014. La fecha de cosecha fue el 2 de abril.

En la Figura 2 se muestran las áreas de cultivo de los años analizados y la ubicación de la torre de micrometeorológica en cada predio cultivado.

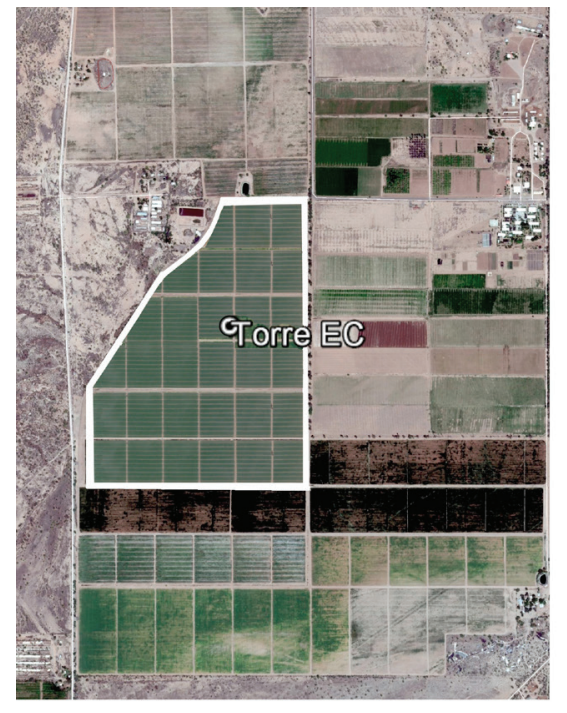

2014

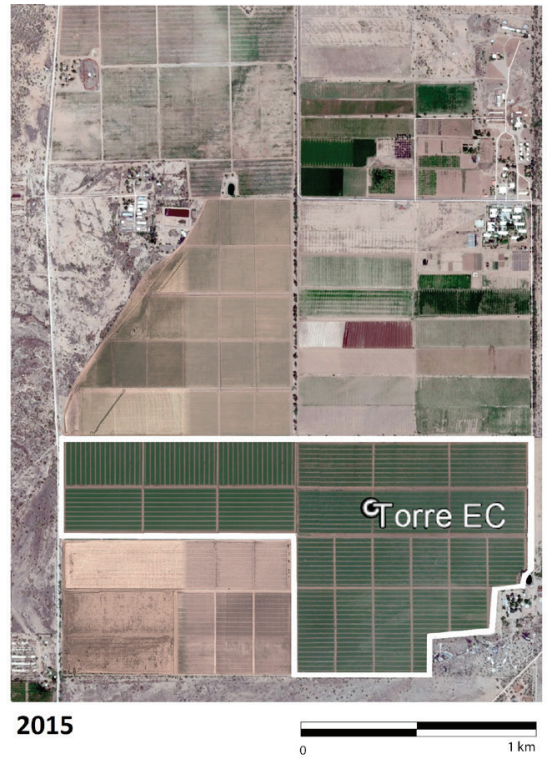

Figura 2. Áreas de cultivo en el sitio de estudio. 


\section{Datos de PROBA-V}

Las imágenes satelitales utilizadas fue PROBA-V S1 TOC de NDVI de $100 \mathrm{~m}$ de resolución corregido por efecto atmosférico. Las imágenes se obtuvieron de la página web http://www.vito-eodata.be/. Las condiciones de nubosidad permitieron elaborar series de trece imágenes del año 2014 y catorce del 2015. Se delimitaron en las imágenes las áreas de cultivo de ambos periodos de estudio, se normalizaron los valores del NDVI y se extrajo de cada imagen el valor del píxel correspondiente a la ubicación de la torre micrometeorológica.

\section{Datos Micrometeorológicos}

En cada predio cultivado se instaló, por el período analizado, una torre instrumentada para medir los componentes de la ecuación de balance de energía: Radiación neta, Rn; flujo de calor latente, LE y sensible, $\mathrm{H}$ y flujo de calor de suelo, G. También se midieron las variables meteorológicas: temperatura, humedad del aire, velocidad y dirección del viento, precipitación, presión barométrica, radiación solar y del suelo, humedad y temperatura. La información recolectada de los flujos (radiativos y turbulentos) fueron muestreados a $10 \mathrm{~Hz}$, para obtener promedios cada $30 \mathrm{~min}$, y los datos meteorológicos se muestrearon a $30 \mathrm{~s}$ y se almacenaron con promedios a cada $30 \mathrm{~min}$.

\section{Medición de la Evapotranspiración (ET ec}

Los flujos turbulentos: Calor sensible $(\mathrm{H})$ y calor latente (LE) fueron medidos con la técnica de covarianza de vórtices o Eddy Covariance (EC) (Baldocchi, 2003).

Los datos crudos se procesaron con el programa EdyPro versión 4.1 (desarrollado por LI-COR) y se obtuvieron promedios cada $30 \mathrm{~min}$, siguiendo los procedimientos propuestos por Burba (2013), se realizaron las correcciones y particiones requeridas, usando el sitio http://www.bgcjena.mpg.de/ MDIwork/ eddyproc/output/20141205021959/results_ext.php. Considerando que el déficit en la ecuación de balance de energía (entrante y saliente) se mantuvo entre el 10 y $20 \%$, no fue necesario aplicar la corrección de la relación de Bowen (Twine et al., 2000) para la obtención de los flujos turbulentos (LE y H). Los datos meteorológicos y de suelo fueron promediados a $30 \mathrm{~min}$ y posteriormente se obtuvieron los valores diarios.
La lámina de evapotranspiración en $\mathrm{mm} \mathrm{d}^{-1}$ se obtuvo haciendo la conversión de unidades considerando que el flujo de calor latente $\left(\mathrm{W} \mathrm{m}^{-2}\right)$ es la energía requerida en el proceso de evapotranspiración por unidad de tiempo y que el calor latente de evaporación $\lambda=2.45 \mathrm{MJ} \mathrm{kg}^{-1}$.

\section{Cálculo de la Evapotranspiración de Referencia}

La evapotranspiración de referencia $\left(\mathrm{Et}_{0}\right)$ se determinó con la ecuación de Penman-Monteith modificada por Allen et al. (1998).

$$
E T_{0}=\frac{0.408 \Delta\left(R_{n}-G\right)+\gamma \frac{900}{T+273} u_{2}\left(e_{s}-e_{a}\right)}{\Delta+\gamma\left(1+0.34 u_{2}\right)}
$$

donde: $\mathrm{ET}_{0}$ es la evapotranspiración de referencia, en

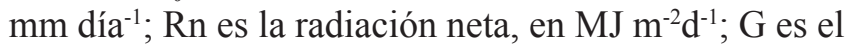
flujo de calor del suelo, en $\mathrm{MJ} \mathrm{m}^{2} \mathrm{~d}^{-1}$; $\mathrm{T}$ es la temperatura del aire promedio diario, en ${ }^{\circ} \mathrm{C} ; \Delta$ es la pendiente de la curva de presión a saturación a la temperatura $\mathrm{T}$, en $\mathrm{kPa}{ }^{\circ} \mathrm{C}^{-1} ; \gamma$ es constante psicrométrica $\left(\mathrm{kPa}^{\circ} \mathrm{C}^{-1}\right)$; $\mathrm{e}_{\mathrm{s}}$ es la presión de saturación del vapor a temperatura $\mathrm{T}$, en $\mathrm{kPa}$; $\mathrm{e}_{\mathrm{a}}$ es la presión de vapor promedio diario, en $\mathrm{kPa}$ $\mathrm{y}_{2}$ es la velocidad de viento promedio diario a $2 \mathrm{~m}$ de elevación sobre el terreno, en $\mathrm{m} \mathrm{s}^{-1}$.

\section{Coeficiente de Cultivo (Kc)}

El coeficiente de cultivo se determinó utilizando la aproximación de Doorenbos y Pruitt (1977) modificada por Allen et al. (1998):

$K c=K c b * K s+K e=\frac{E T_{c}}{E T_{0}}$

donde: $\mathrm{ET}_{\mathrm{c}}$ es la evapotranspiración medida en $\left(\mathrm{mm} \mathrm{d}^{-1}\right)$ con el método de $\mathrm{EC}, \mathrm{Kcb}$ es la transpiración del cultivo, Ke la evaporación del suelo, Kc el coeficiente de cultivo y Ks el coeficiente de estrés hídrico.

En ambos ciclos, debido a que el cultivo se encontraba sin estrés hídrico, se tomó un valor igual a la unidad para el coeficiente Ks. De igual forma por lo complejo que resulta medir la transpiración y evaporación por separado, se consideró Kc como el cociente entre ETc y ET ${ }_{0}$. 
$K c=\frac{E T_{c}}{E T_{0}}$

\section{Correlación de Ke y NDVI Obtenido con Imágenes de PROBA-V}

Los valores de Kc estimados para el cultivo de sandía del año 2015, se correlacionaron con los valores de NDVI elaborados con las imágenes de PROBA-V del mismo año (Figura 3), obteniéndose un coeficiente de correlación de 0.567 .

\section{Modelo Lineal para Obtención de Kc y ET}

Esta correlación, permitió determinar valores de Kc en función de NDVI con la relación:

$\mathrm{Kc}=0.765 \mathrm{NDVI}+0.295$

Y los valores de ET con la relación:

$\mathrm{ET}=(0.765 \mathrm{NDVI}+0.295) \mathrm{ET}_{0}$

\section{RESULTADOS Y DISCUSIÓN}

En el Cuadro 1 se muestran, para los trece días de análisis del cultivo de sandía del año 2014, los valores de evapotranspiración (ET) medidos con el método de

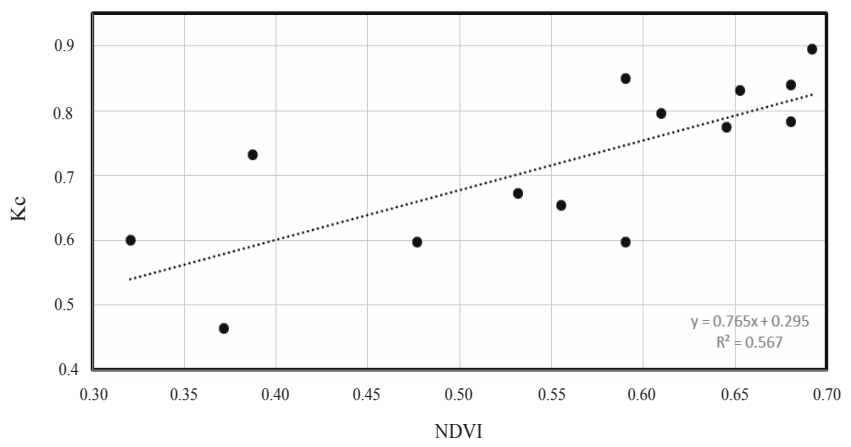

Figura 3. Comportamiento del coeficiente de cultivo (Kc) en relación al NDVI obtenido con imágenes de PROBA-V.

covarianza de vórtices, $\mathrm{ET}_{0}$ estimado con la ecuación de Penman-Monteith, NDVI obtenido de las imágenes de PROBA-V, los valores de Kc y los de ET estimados con el modelo Elaborado.

En la Figura 4, se muestra la comparación entre los valores medidos de ET y los estimados con PROBA-V, y como lo indica el coeficiente de correlación $(r=0.729)$ el modelo lineal estima con precisión aceptable la evapotranspiración en el cultivo de sandía, aunque sobreestima la lámina de agua evapotranspirada durante todo el cultivo de sandía analizado (Figura 5). En la misma figura, también, se puede apreciar que la variabilidad temporal que muestra la ET estimada con las imágenes de PROBA-V es congruente con las fluctuaciones de la variable medida.

Cuadro 1. Estimación de la evapotranspiración para el año 2014.

\begin{tabular}{|c|c|c|c|c|c|c|}
\hline \multicolumn{7}{|c|}{ - - - mm día ${ }^{-1} \ldots$} \\
\hline 14-Sep-14 & 257 & 3.93 & 6.99 & 0.44 & 0.63 & 4.40 \\
\hline 23-Sep-14 & 266 & 3.54 & 6.17 & 0.63 & 0.77 & 4.78 \\
\hline 11-Oct-14 & 284 & 3.06 & 5.56 & 0.64 & 0.78 & 4.35 \\
\hline 16-Oct-14 & 289 & 2.42 & 6.14 & 0.62 & 0.77 & 4.71 \\
\hline 20-Oct-14 & 293 & 1.61 & 3.85 & 0.58 & 0.74 & 2.85 \\
\hline 25-Oct-14 & 298 & 2.02 & 4.64 & 0.62 & 0.77 & 3.57 \\
\hline 12-Nov-14 & 316 & 1.05 & 3.22 & 0.28 & 0.51 & 1.65 \\
\hline
\end{tabular}




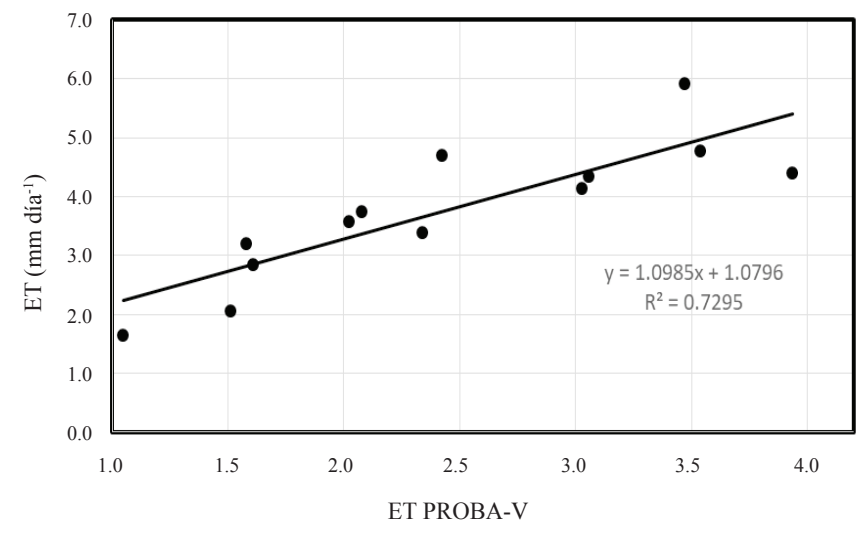

Figura 4. Comparación de evapotranspiración (ET) medida en el sitio con los valores estimados con PROBA-V.

Los resultados reflejan el comportamiento de los elementos del tiempo atmosférico prevaleciente durante el ciclo de cultivo y condicionan los valores determinados con el método de Flujo turbulento y los estimados con las imágenes de PROBA-V. En las tres semanas posteriores a la primera semana de trasplante, se registraron $195 \mathrm{~mm}$ de lluvia, de los 292 registrados durante el ciclo de cultivo (Figura 6), propiciando la aparición de malezas que contribuyeron a las láminas de evapotranspiración medidas con el método de covarianza de vórtices. La radiación solar decreció desde el inicio del cultivo con notables disminuciones durante los días con lluvia o nublados que limitaron la visibilidad del sensor de PROBA-V y la cantidad de imágenes de NDVI utilizadas para el estudio.

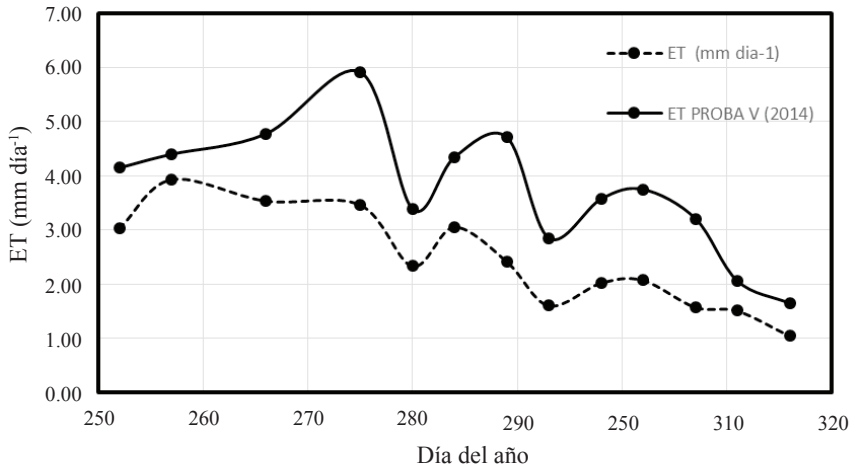

Figura 5. Comportamiento de evapotranspiración (ET) medida y ET estimada con PROBA-V en 2014.

La evapotranspiración total en todo el período de cultivo medida con el método de flujo turbulento en el sitio de estudio fue $214.4 \mathrm{~mm}$, superior a los $180 \mathrm{~mm}$ cuantificados con el mismo procedimiento por Román et al. (2017), pero dentro del rango de $250 \mathrm{~mm} \mathrm{a}$ $350 \mathrm{~mm}$ medidos con lisímetro reportados por Erdem et al. (2005), Bastos et al. (2012) y Shukla et al. (2014). En el período analizado, limitado por la disponibilidad de imágenes, la estimación con PROBA-V fue de $255.22 \mathrm{~mm}, 86.57 \mathrm{~mm}$ por encima de la medida en la torre micrometeorológica en el mismo período.

En cuanto al coeficiente de cultivo $(\mathrm{Kc})$, los valores obtenidos durante los días intermedios, coinciden con el promedio semanal máximo $\mathrm{Kc}=0.73$ obtenido por Román et al. (2017).

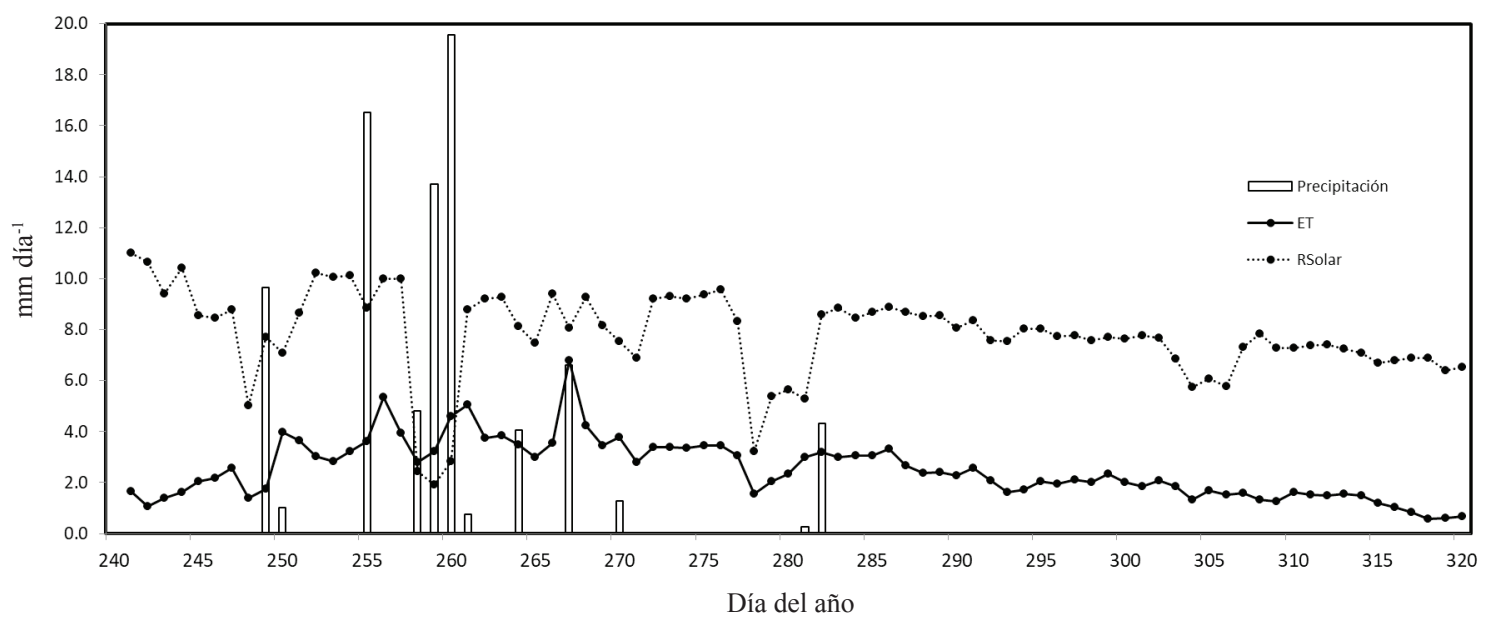

Figura 6. Variabilidad temporal de la precipitación, la radiación solar y la evapotranspiración durante el período de análisis en el sitio de estudio en 2014. 
El modelo lineal se elaboró a partir de los datos del año 2015, en el que el ciclo del cultivo abarcó los meses de enero a abril, en los que la correlación entre Kc y NDVI fue de $\mathrm{r}^{2}=0.567$ con una serie de 14 imágenes de NDVI, que a diferencia del año 2014, en el que el cultivo se desarrolló de agosto a noviembre, solo se obtuvieron 13 imágenes libres de nubes, obteniéndose una correlación de $\mathrm{r}^{2}=0.528$.

\section{CONCLUSIONES}

- Este estudio muestra que es posible estimar evapotranspiración con imágenes de NDVI obtenidas con PROBA-V y el error medio cuadrático EMC = $1.44 \mathrm{~mm} \mathrm{día}^{-1}$ de los valores estimados con respecto a los medidos, que en los días de mayor evapotranspiración representan una sobreestimación cercana al $12 \%$ y en los días de menor evapotranspiración alcanza el 57\%, se puede reducir con más medidas de evapotranspiración y más imágenes de satélite del área de estudio durante los dos ciclos de cultivo de sandía y elaborando modelos independientes para cultivos de primavera y verano, en las que se consideren las variables climáticas propias de cada estación en la región, donde es necesario hacer un uso más eficiente del agua.

- El estudio, también muestra, que se mantiene el legado del sensor Vegetation que opera desde 1998 a bordo de los satélites SPOT 4 y SPOT 5 y estudios futuros pueden enfocarse, a partir de datos de estaciones micrometeorlógicas, a explicar el efecto de las variables climatológicas de la diferencia entre los valores medidos de ET y los estimados con PROBA-V de manera que se determinen los factores de escala que permitan mayor precisión en la estimación con sensores remotos de esta variable hidroclimática.

\section{AGRADECIMIENTOS}

Al Consejo Nacional de Ciencia y Tecnología, del gobierno de la República Mexicana por su apoyo para realizar estudios de posgrado.

\section{LITERATURA CITADA}

Allen, R. G., L. S. Pereira, D. Raes, and M. Smith. 1998. Crop evapotranspiration - Guidelines for computing crop water requirements - FAO Irrigation and drainage paper 56. Rome, Italy. ISBN 92-5-104219-5.

Allen, R. G. 2000. Using the FAO-56 dual crop coefficient method over an irrigated region as part of an evapotranspiration intercomparison study. J. Hydrol. 229: 27-41.

Allen, R. G., L. S. Pereira, D. Raes, and M. Smith. 2006. Evapotranspiración de cultivo: Guías para la determinación de los requerimientos de agua de los cultivos. Estudio FAO riego y drenaje No. 56, Roma, Italia.

Baldocchi, D. D. 2003. Assessing the eddy covariance technique for evaluating carbon dioxide exchange rates of ecosystems: Past, present and future. Global Changes Biol. 9: 479 -492

Bastos, A. E., C. R. Silva, B. H. N. Rodrigues, A. S. Andrade, and L. M. M. Ibiapina. 2012. Evapotranspiration and crop coefficient of drip irrigated watermelon in Piaui coastline, Brazil. Eng. Agric. 32: 582-590.

Batra, N., S. Islam, V. Venturini, G. Bisht, and L. Jiang. 2006. Estimation and comparison of evapotranspiration from MODIS and AVHRR sensors for clear sky days over the Southern Great Plains. Remote Sens. Environ. 103: 1-15.

Bisquert, M., J. M. Sánchez, R. López-Urrea, V. Caselles, and J. Galve. 2016. Downscaling LST data to estimate field-scale evapotranspiration using images from the SPOT-5 Take-5 experiment. Living planet symposium 2016. European Space Agency. Prague, Czech Republic.

Brutsaert, W. 1982. Evaporation into the atmosphere: Theory, history and applications. Springer Netherlands. Dordrecht, Netherlands.

Burba, G. 2013. Eddy covariance method for scientific, industrial, agricultural, and regulatory applications. A field book on measuring ecosystem gas exchange and areal emission rates. LI-COR Biosciences. Lincoln, NE, USA.

Dierckx, W., S. Sterckx, I. Benhadj, S. Livens, G. Duhoux, T. Van Achteren, M. Francois, K. Mellab, and G. Saint. 2014. PROBA-V Mission for global vegetation monitoring: Standard products and image quality. Int. J. Remote Sens. 35: 2589-2614.

Doorenbos, J. and W. O. Pruitt. 1977. Guidelines for predicting crop water requirements. FAO irrigation and drainage paper 24. FAO. Rome, Italy.

Erdem, Y., T. Erdem, A. Orta, and H. Okursoy. 2005. Irrigation scheduling for watermelon with crop water stress index (CWSI). J. Central Eur. Agric. 6: 449-460.

Farg, E., S. M. Arafat, M. S. Abd El-Wahed, and A. M. ElGindy. 2012. Estimation of evapotranspiration ETc and crop coefficient Kc of wheat, in south Nile Delta of Egypt using integrated FAO-56 approach and remote sensing data. The Egyptian J. Remote Sens. Space Sci. 15: 83-89. 
Francois, M., S. Santandrea, K. Mellab, D. Vrancken, and J. Versluys. 2014. The PROBA-V mission: The space segment. Int. J. Remote Sens. 35: 2548-2564.

Jiang, L. and S. Islam. 1999. A methodology for estimation of surface evapotranspiration over large areas using remote sensing observations. Geophys. Res. Lett. 26: 2773-2776.

Ma, Y., Z. Zhu, L. Zhong, B. Wang, C. Han, Z. Wang, Y. Wang, L. Lu, P. M. Amatya, W. Ma, and Z. Hu. 2014. Combining MODIS, AVHRR and in situ data for evapotranspiration estimation over heterogeneous landscape of the Tibetan Plateau. Atmos. Chem. Phys. 14: 1507-1515.

Méndez-Barroso, L.A., E. R. Vivoni, A. Robles-Morua, G. Mascaro, E. A. Yépez, J. C. Rodríguez, C. J. Watts, J. Garatuza-Payán, and J. A. Saiz-Hernández. 2014. A modeling approach reveals differences in evapotranspiration and its partitioning in two semiarid ecosystems in Northwest Mexico. Water Resour. Res. 50: 3229-3252.

Parlange, M. B., W. E. Eichinger, and J. D. Albertson. 1995. Regional scale evaporation and the atmospheric boundary layer. Rev. Geophys. 33: 99-124.

Polhamus, A., J. B. Fisher, and K. P. Tu. 2013. What controls the error structure in evapotranspiration models? Agric. For. Meteorol. 169: 12-24.

Rodríguez, J. C., J. Grageda, C. J. Watts, J. Garatuza-Payán, A. Castellanos-Villegas, J. Rodríguez-Casas, J. A. SaizHernández, and V. Olavarrieta. 2010. Water use by perennial crops on the lower Sonora watershed. J. Arid Environ. 74: 603-610.

Román-Román, L., T. Díaz-Valdés, E. López-Avendaño, C. Watts, F. Cruz-Bautista, J. Rodríguez-Casas y J. C. Rodríguez.
2017. Evapotranspiración del cultivo de sandía (Citrullus lantanus) en la Costa de Hermosillo, Sonora, México. Terra Latinoamericana 35: 41-49.

Senay, G. B., M. Friedrichs, R. K. Shing, and N. M. Velpuri. 2016. Evaluating Landsat 8 evapotranspiration for water use mapping in the Colorado River Basin. Remote Sens. Environ. 185: 171-185.

Shukla, S., N. K. Shrestha, F. H. Jaber, S. Srivastava, T. A. Obreza, and B. J. Boman. 2014. Evapotranspiration and crop coefficient for watermelon grown under plastic mulched conditions in sub-tropical Florida. Agric. Water Manage. 132: 1-9.

Shuttleworth, W. J. 2007. Putting the "vap" into evaporation. Hydrol. Earth System Sci. 11: 210-244.

Twine, T. E., W. P. Kustas, J. M. Norman, D. R. Cook, P. R. Houser, T. P. Meyers, J. H. Prueger, P. J. Starks, and M. L. Wesely. 2000. Correcting eddy-covariance flux underestimates over a grassland. Agric. For. Meteorol. 103: 279-300.

Vivoni, E. R., J. C. Rodríguez and C. J. Watts. 2010. On the spatiotemporal variability of soil moisture and evapotranspiration in a mountainous basin within the North American monsoon region. Water Resour. Res. 46: W02509. doi: 10.1029/2009WR008240

Watts, C. J., J. C. Rodríguez, J. Garatuza-Payán, H. de Bruin y J. Stewart. 1999. Estimación de evaporación y radiación solar en el valle del Yaqui Sonora usando datos de satélite. Ing. Hidrául. Méx. 14: 45-53.

Yang, Y., M. C. Anderson, F. Gao, C. R. Hain, K. A. Semmens, W. P. Kustas, A. Noormets, R. H. Wynne, V. A. Thomas, and G. Sun. 2017. Daily Landsat-scale evapotranspiration estimation over a forested landscape in North Carolina, USA, using multisatellite data fusion. Hidrol. Earth Syst. Sci. 21: 1017-1037. 